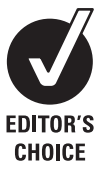

CHOICE

\title{
Prognostic value of coronary revascularisation- related myocardial injury: a cardiac magnetic resonance imaging study
}

\author{
K Rahimi, ${ }^{1}$ A P Banning, ${ }^{2}$ A S H Cheng, ${ }^{3}$ T J Pegg, ${ }^{4}$ T D Karamitsos, ${ }^{4}$ K M Channon, ${ }^{2}$ \\ S Darby, ${ }^{1}$ D P Taggart, ${ }^{5}$ S Neubauer, ${ }^{6}$ J B Selvanayagam ${ }^{7}$
}

${ }^{1}$ University of Oxford, Clinical Trial Service Unit and Epidemiological Studies Unit, Oxford, UK; ${ }^{2}$ John Radcliffe Hospital, Department of Cardiology, Oxford, UK:

${ }^{3}$ University of Oxford, Department of Cardiovascular Medicine, Centre for Clinical Magnetic Resonance Research, Oxford, UK; ${ }^{4}$ University of Oxford, Department of Cardiovascular Medicine, Oxford, UK; ${ }^{5}$ University of Oxford, Nuffield Department of Surgery, Oxford, UK; ${ }^{6}$ The University of Oxford Centre for Clinical Magnetic Resonance Research, Oxford, UK; ${ }^{7}$ Flinders University of South Australia, Flinders Medical Centre, Department of Cardiovascular Medicine, Adelaide, Australia

Correspondence to: Professor Joseph Selvanayagam, Flinders University of South Australia, Director Cardiac MR and CT, Flinders Medical Centre, Adelaide 5042, Australia; joseph.selvanayagam@flinders. edu.au

SN and JBS are joint senior authors

Accepted 4 August 2009

Published Online First

16 August 2009

\section{ABSTRACT}

Aims: Myocardial revascularisation improves outcomes in patients with coronary artery disease. However, these procedures may themselves cause irreversible myocardial injury. The prognostic value of procedural myocardial injury is uncertain.

Methods and results: We quantified procedural myocardial necrosis using delayed enhancement cardiovascular magnetic resonance imaging (DE-CMR) in 152 consecutive patients before and shortly after percutaneous coronary intervention (PCI) or coronary artery bypass graft (CABG). The primary endpoint was defined as death, non-fatal myocardial infarction, sustained ventricular arrhythmia, unstable angina or heart failure requiring hospitalisation. During a median follow-up of 2.9 years, 27 patients $(18 \%)$ reached the primary endpoint. 49 patients (32\%) had evidence of new procedure-related myocardial hyperenhancement with a median mass of $5.0 \mathrm{~g}$ (interquartile range 2.7-9.8). After adjustment for age and sex, these patients had a 3.1-fold $195 \%$ confidence interval 1.4 to 6.8; $p=0.004$ ) higher risk of adverse outcome than patients without new hyperenhancement. Cardiac troponin levels and quantitative measures of left ventricular function after procedure did not show any significant independent association with the primary endpoint and they did not alter the independent association of new hyperenhancement.

Conclusions: Myocardial injury during $\mathrm{PCl}$ or $\mathrm{CABG}$, identified by DE-CMR, adversely affects clinical outcome. This suggests the benefits from revascularisation could partially be offset by new myocardial injury caused by the intervention itself.

A rise in cardiac biomarkers occurs in nearly all patients undergoing coronary artery bypass graft (CABG) surgery ${ }^{1}$ and in up to $40 \%$ of patients undergoing percutaneous coronary intervention (PCI). ${ }^{2}$ The most recent joint European Society of Cardiology (ESC)/American College of Cardiology (ACC)/American Heart Association (AHA)/World Heart Federation (WHF) task force guidelines recognise elevation of cardiac biomarkers of more than three times the 99th percentile upper reference limit (URL) following PCI and more than five times the 99th percentile URL after CABG in the definition of procedure-related myocardial infarction. ${ }^{3}$ However, controversy remains over the independent prognostic value of rises in biomarkers after revascularisation. ${ }^{45}$

Although biomarkers such as troponin have a high accuracy in detection of myocardial tissue loss and have been shown to be of prognostic value in several acute and non-acute settings, the demonstration of their prognostic value after coronary revascularisation has been hampered for several reasons: troponin elevations after revascularisation are often very small and the cut-offs for abnormal values variable across studies; many patients undergoing elective revascularisation have some degree of troponin elevation before the procedure $^{67}$; the inherent delay in rise of biomarkers may affect the correct ascertainment of sequence of events, ${ }^{8}$ in particular when revascularisation itself can lead to biomarker release from damaged areas that become reperfused ${ }^{9}$; and rise in values might be confounded by other patient-related or procedure-related factors. ${ }^{2}$ These complexities may explain the inconsistencies in previous reports from studies that have assessed the prognostic value of procedural myocardial injury with the use of biomarkers.

Delayed enhancement cardiac magnetic resonance imaging (DE-CMR) allows assessment of the transmural extent of irreversible injury (seen as hyperenhancement), and is superior to single photon emission computed tomography (SPECT) for the identification of subendocardial myocardial infarction. ${ }^{10-13}$ DE-CMR permits precise quantification of even small areas of myocardial necrosis, such as those occurring after surgical or percutaneous revascularisation. ${ }^{14-16}$ Although evidence exists from other clinical settings that presence of small areas of hyperenhancement (approximately $2 \%$ of left ventricular mass) is associated with poor clinical outcome, ${ }^{17}$ its relevance after revascularisation remains uncertain.

In the present study, we evaluated the prognostic importance of procedure-related myocardial injury, quantified by DE-CMR, in a consecutive series of patients undergoing revascularisation by either PCI or CABG. We hypothesised that both the presence and magnitude of new irreversible injury would have prognostic value beyond standard clinical, biochemical and functional measures of myocardial injury.

\section{METHODS}

\section{Patient population and CMR imaging time points}

The study constitutes the mid-term follow-up of 152 patients, 92 undergoing complex PCI and 60 undergoing $\mathrm{CABG}$. The cross-sectional reports investigating the association between troponin release after PCI or CABG and new myocardial hyperenhancement in 115 of these patients with the full inclusion and exclusion criteria have 
previously been published. ${ }^{14}{ }^{15}$ Briefly, PCI patients were those with either one-vessel or two-vessel coronary artery disease scheduled for complex intervention (30 $\mathrm{mm}$ of stent to a single vessel or treatment of a segment that involved at least one major side branch $>2.0 \mathrm{~mm}$ in size and patients undergoing planned two-vessel $\mathrm{PCI}$ ) and CABG patients were those undergoing first time surgery excluding concomitant valve surgery. The indication for revascularisation was stable angina in 139 patients and non-ST elevation acute coronary syndrome (during the preceding 4 weeks) in the remaining 13 patients. We excluded patients with typical magnetic resonance imaging (MRI) contraindications. Patients were imaged usually 24 hours before and early after the intervention (24 hours post PCI, median 6 days post CABG).

\section{CMR protocol}

Patients were studied in a $1.5 \mathrm{~T}$ clinical magnetic resonance scanner (Siemens Sonata, Erlangen, Germany), and steady state free precession cine images were acquired in two long-axis, and seven to nine short-axis views, as previously described. ${ }^{14} \mathrm{~A}$ gadolinium-based contrast agent (Gadodiamide, Omniscan, Nycomed Amersham) was then administered intravenously at a dose of $0.100-0.125 \mathrm{mmol} / \mathrm{kg}$ of body weight. Delayed enhancement images were acquired after an 8 minute delay with the use of an inversion-recovery segmented gradient echo sequence as previously described. ${ }^{14} 18$

\section{CMR post processing and data analysis}

For the analysis of global left ventricular (LV) function, the following variables were determined by planimetry of all the short axis cine images: LV end-diastolic volume index (LVEDVI), LV end-systolic volume index (LVESVI), LV ejection fraction (LVEF) $(\%)$ and LV mass index $\left(\mathrm{g} / \mathrm{m}^{2}\right)$. Areas of late gadolinium-DTPA hyperenhancement were quantified using computer-assisted planimetry on each of the short-axis images, by an observer without knowledge of the cine CMR, procedural or biochemical findings. Hyperenhanced pixels were defined as those with image intensities greater than two standard deviations above the mean of image intensities in a remote myocardial region in the same image. Pre-intervention and postintervention scans were read side by side. The method of analysing and calculating mass of hyperenhancement is standardised within our unit and the reproducibility has been published. ${ }^{19}$

\section{Clinical follow-up and definition of endpoints}

The primary study endpoint was pre-specified as the earliest occurrence of death, non-fatal myocardial infarction, admission to hospital for unstable angina or worsening heart failure, or occurrence of ventricular arrhythmia (defined as ventricular fibrillation or sustained ventricular tachycardia). All non-fatal components of this composite endpoint have been shown to confer a poor prognosis and they also have a plausible causative link to new myocardial tissue loss. However, in order to ensure that the symptom-driven events, which are relatively common, were not the chief determinants of any observed association, we also pre-specified a secondary endpoint that excluded admission for unstable angina or heart failure. This secondary endpoint was therefore defined as the occurrence of death, non-fatal myocardial infarction or ventricular arrhythmia only.

Information about outcomes of interest was sought primarily from patients by telephone interview using a standard followup questionnaire. We contacted patients' general physicians by mail or telephone when complete follow-up information was not available from the patients themselves. For the remaining patients for whom no clinical follow-up data were available, information about survival status was sought from the national death registers.

We obtained complete follow-up information for 140 patients and had information about the vital status in another 11 patients. Only one patient was lost to follow-up. All data were collected and coded by staff blinded to clinical, biochemical and CMR results.

\section{Biochemistry}

Serum was collected from each patient for measurement of cardiac troponin I (troponin) before and 24 hours after PCI or CABG. All troponin samples were analysed using an immunoassay analyser (Immulite; Diagnostic Products Corporation, Wales, UK). The lower limit of quantification and the 99th centile of URL was $0.2 \mu \mathrm{g} / \mathrm{l}$, and the upper limit of the normal in our laboratory was $1.0 \mu \mathrm{g} / \mathrm{l}$. The assay precision, represented by the percentage coefficient of variation, was $8.4 \%$ at $0.8 \mu \mathrm{g} / \mathrm{l}$, $7.6 \%$ at $8.0 \mu \mathrm{g} / \mathrm{l}$ and $7.6 \%$ at $86 \mu \mathrm{g} / 1 .{ }^{20}$

\section{Statistical analysis}

Patients were classified into two groups based on presence or absence of new hyperenhancement after revascularisation. To account for measurement errors that occur in the processing of areas of hyperenhancement, we set the cut-off point to determine the presence or absence of new hyperenhancement as $2 \mathrm{~g}$ when there were cases of pre-existing hyperenhancement, or, if the new hyperenhancement was in a previously unaffected area, no limit was placed. ${ }^{19}$ Demographic variables, clinical characteristics, coronary angiogram and CMR findings in the two groups were compared using the Student $t$ test for continuous variables and a $\chi^{2}$ test for categorical variables (table 1). Then, to identify potential predictors of the outcome, we first performed a univariate Cox-regression for all variables with time to first occurrence of the endpoint as the dependent variable (table 2). In a second step, all variables that were significantly associated with an increased hazard ratio in the univariate analysis were entered as covariates into a sexadjusted and age-adjusted multiple Cox regression. Variables not showing an independent association with the outcome were subsequently removed from the model one by one (least significant first) until only significant variables remained. Finally, we added each of the other variables from table 2 into the model in turn to assess their marginal significance in the regression and their impact on the predictive value of the significant variables (table 3 ). The above analyses were performed separately for new hyperenhancement as a categorical and as a continuous variable. Confidence intervals were checked using bootstrapping and found to be only slightly wider than those based on the standard asymptotic theory. Ageadjusted and sex-adjusted event-free survival estimates were compared by Kaplan-Meier survival curves for patients with and without new hyperenhancement. For all analyses, a two-sided $p$ value of less than or equal to 0.05 was considered significant. The Stata statistics package (Version 9) was used for all analyses.

\section{RESULTS}

We found evidence of new revascularisation-related hyperenhancement in $32 \%$ of the patients (49 out of 152), which was significantly lower than if the cut-offs for troponin according to 
Table 1 Demographic variables, clinical characteristics and coronary angiogram and cardiovascular magnetic resonance imaging measures in patients with or without procedure-related hyperenhancement

\begin{tabular}{|c|c|c|c|}
\hline & \multicolumn{2}{|c|}{ New hyperenhancement } & \multirow{2}{*}{$\begin{array}{l}\text { p Value for } \\
\text { difference }\end{array}$} \\
\hline & Absent $(n=103)$ & Present $(n=49)$ & \\
\hline \multicolumn{4}{|l|}{ Demographic variables: } \\
\hline Age (years) & $64(9)^{*}$ & $63(13)^{*}$ & 0.29 \\
\hline Male (\%) & 73 & 74 & 0.93 \\
\hline \multicolumn{4}{|l|}{ Clinical characteristics: } \\
\hline Hypertension (\%) & 73 & 61 & 0.16 \\
\hline Diabetes (\%) & 25 & 20 & 0.49 \\
\hline Hypercholesterolaemia (\%) & 77 & 73 & 0.59 \\
\hline Current smoker (\%) & 34 & 43 & 0.31 \\
\hline History of MI (\%) & 25 & 39 & 0.09 \\
\hline Presentation with ACS (\%) & 7 & 12 & 0.26 \\
\hline $\mathrm{PCl}$ cohort (\%) & 65 & 51 & 0.10 \\
\hline Troponin before revascularisation $(\mu \mathrm{g} / \mathrm{l})$ & $0.0(0.0-0.0) \dagger$ & $0.0(0.0-0.0) \dagger$ & 0.75 \\
\hline Troponin after revascularisation $(\mu \mathrm{g} / \mathrm{l})$ & $0.2(0.0-1.1) \dagger$ & $1.9(1.2-5.2) \dagger$ & 0.03 \\
\hline PMI (\%) & 29 & 89 & $<0.001$ \\
\hline \multicolumn{4}{|l|}{ Medication (\%): } \\
\hline Anti-platelets & 95 & 95 & 1.00 \\
\hline Statin & 95 & 100 & 0.14 \\
\hline ACE inhibitor & 82 & 84 & 0.72 \\
\hline$\beta$-blocker & 73 & 68 & 0.61 \\
\hline Calcium channel blocker & 27 & 32 & 0.61 \\
\hline \multicolumn{4}{|l|}{ Coronary angiogram $(\%)$ : } \\
\hline 1-vessel disease & 40 & 25 & $0.18 \%$ \\
\hline 2-vessel disease & 30 & 37 & \\
\hline 3-vessel disease & 30 & 39 & \\
\hline Presence of TCO & 20 & 20 & 0.99 \\
\hline \multicolumn{4}{|l|}{ CMR before revascularisation: } \\
\hline LVEF (\%) & $65(13)^{*}$ & $61(12)^{*}$ & 0.10 \\
\hline LVEDVI (ml) & $78(20)^{*}$ & $83(26)^{*}$ & 0.21 \\
\hline LVESVI (ml) & $29(18)^{*}$ & $34(24)^{*}$ & 0.09 \\
\hline SVI (ml) & $50(11)^{*}$ & $48(8)^{*}$ & 0.46 \\
\hline LV mass index $(\mathrm{g})$ & $64(14)^{*}$ & $64(15)^{*}$ & 0.79 \\
\hline LV mass absolute $(\mathrm{g})$ & $127(33)^{*}$ & $123(32)^{*}$ & 0.42 \\
\hline Hyperenhancement (g) & $0(0-9.6) \dagger$ & $5.6(0-15.7) \dagger$ & 0.14 \\
\hline \multicolumn{4}{|l|}{ CMR after revascularisation: } \\
\hline LVEF $(\%)$ & $66(12)^{*}$ & $61(13)^{*}$ & 0.02 \\
\hline Change in LVEF pre/post (\%) & $1.2(6.7)^{*}$ & $-0.5(8.5)^{*}$ & 0.21 \\
\hline LVEDVI (ml) & $77(18)^{*}$ & $85(28)^{*}$ & 0.05 \\
\hline LVESVI (ml) & $28(16)^{*}$ & $36(26)^{*}$ & 0.02 \\
\hline SVI (ml) & $50(10)^{*}$ & $50(15)^{*}$ & 0.75 \\
\hline LV mass index $(\mathrm{g})$ & $63(13)^{*}$ & $67(16)^{*}$ & 0.14 \\
\hline LV mass absolute (g) & $126(31)^{*}$ & $128(35)^{*}$ & 0.72 \\
\hline Hyperenhancement (g) & $0.0(0.0-9.4) \dagger$ & $12.5(6.0-20.8) \dagger$ & $<0.001$ \\
\hline \multicolumn{4}{|c|}{$\begin{array}{l}\text { *Mean (SD). } \\
\text { †Median (interquartile range). } \\
\text { \$Test for trend across the three groups. } \\
\text { ACE, angiotensin-converting enzyme; ACS, acute coronary syndrome; CMR, cardiovascular magnetic resonance imaging; LVEF, left } \\
\text { ventricular ejection fraction; LVEDVI, left ventricular end-diastolic volume index; LVESVI, left ventricular end-systolic volume index; } \\
\text { LV, left ventricle; MI, myocardial infarction; PCI, percutaneous coronary intervention; PMI, procedure-related myocardial infarction } \\
\text { as detected by troponin }>3 \times \text { or } 5 \times \text { the } 99 \text { th centile of the upper range of limit for PCI and CABG, respectively according to the } \\
\text { ESC/ACC/AHA/WHF guidelines; SVI, stroke volume index; TCO, total chronic coronary occlusion. }\end{array}$} \\
\hline
\end{tabular}

the ESC/ACC/AHA/WCF guidelines regarding peri-procedural myocardial infarction ${ }^{3}$ (that is, elevation more than three times the 99th percentile URL following PCI and more than five times after CABG) were used $(50 \%, \mathrm{p}<0.001)$. The median new myocardial injury measured by DE-CMR was relatively small (5.0 g, interquartile range (IOR) 2.7-9.8). Patients' characteristics before the intervention, including the findings from CMR and coronary angiogram, were similar in those with and without evidence of new hyperenhancement (table 1). Fourteen patients $(9 \%)$ had small rises in troponin before revascularisation (median $0.85 \mu \mathrm{g} / \mathrm{l}$ ). As expected, patients who developed new procedure-related hyperenhancement were also more likely to have raised troponin and evidence of LV functional impairment after coronary intervention (lower LVEF and higher LVESVI).

Median follow-up duration was 2.9 years (IOR 0.9-4.9). During the observation period, 27 patients $(18 \%)$ reached the primary endpoint (three deaths, five non-fatal myocardial infarctions, two ventricular arrhythmias, four admissions to hospital with unstable angina and 13 admissions due to worsening heart failure). Two patients with worsening heart failure died subsequently, so that 12 patients $(8 \%)$ reached the 
Table 2 Prognostic value of demographic variables, clinical characteristics and findings from coronary angiogram and cardiovascular magnetic resonance imaging in the univariate analysis

\begin{tabular}{|c|c|c|c|c|}
\hline & \multicolumn{2}{|l|}{ Primary endpoint* } & \multicolumn{2}{|l|}{ Secondary endpoint $\dagger$} \\
\hline & HR (95\% Cl) & p Value & HR (95\% Cl) & p Value \\
\hline \multicolumn{5}{|l|}{ Demographic variables: } \\
\hline Age (years) & $1.05(1.01$ to 1.09$)$ & 0.007 & $1.04(0.98$ to 1.10$)$ & 0.23 \\
\hline Sex (M) & $0.79(0.27$ to 2.30$)$ & 0.67 & $1.67(0.45$ to 6.22$)$ & 0.45 \\
\hline \multicolumn{5}{|l|}{ Clinical characteristics: } \\
\hline Hypertension $(\mathrm{y} / \mathrm{n})$ & $0.98(0.43$ to 2.25$)$ & 0.97 & $1.20(0.32$ to 4.43$)$ & 0.79 \\
\hline Diabetes $(\mathrm{y} / \mathrm{n})$ & $0.97(0.39$ to 2.40$)$ & 0.94 & $1.74(0.52$ to 5.80$)$ & 0.37 \\
\hline Hypercholesterolaemia (y/n) & 1.51 (0.61 to 3.77$)$ & 0.37 & $0.94(0.28$ to 3.15$)$ & 0.92 \\
\hline Current smoker $(\mathrm{y} / \mathrm{n})$ & $0.86(0.39$ to 1.92$)$ & 0.72 & $1.68(0.54$ to 5.23$)$ & 0.37 \\
\hline History of $\mathrm{Ml}(\mathrm{y} / \mathrm{n})$ & $1.44(0.67$ to 3.11$)$ & 0.35 & $2.09(0.67$ to 6.47$)$ & 0.20 \\
\hline Presentation with ACS $(\mathrm{y} / \mathrm{n})$ & $0.74(0.17$ to 3.14$)$ & 0.68 & $1.98(0.42$ to 9.35$)$ & 0.39 \\
\hline $\mathrm{PCl}$ cohort $(\mathrm{y} / \mathrm{n})$ & $0.83(0.37$ to 1.87$)$ & 0.66 & $0.38(0.10$ to 1.50$)$ & 0.17 \\
\hline Troponin before revascularisation $(\mu \mathrm{g} / \mathrm{l})$ & $0.47(0.08$ to 2.73$)$ & 0.40 & $1.0(0.19$ to 5.19$)$ & 1.0 \\
\hline Troponin after revascularisation $(\mu \mathrm{g} / \mathrm{l})$ & $1.02(0.97$ to 1.07$)$ & 0.38 & $0.98(0.88$ to 1.09$)$ & 0.72 \\
\hline PMI & $2.23(0.91$ to 5.93$)$ & 0.08 & $2.97(0.62$ to 14.21$)$ & 0.17 \\
\hline Troponin $>0.2 \mu \mathrm{g} / \mathrm{t}$ & $2.09(0.62$ to 7.05$)$ & 0.24 & $2.66(0.34$ to 21.06$)$ & 0.36 \\
\hline \multicolumn{5}{|l|}{ Medication (y/n): } \\
\hline Anti-platelets & $-\boldsymbol{\oplus}$ & 1.00 & $-\boldsymbol{\top}$ & 1.00 \\
\hline Statin & $-\Phi$ & 1.00 & $-\Phi$ & 1.00 \\
\hline ACE inhibitor & $1.72(0.39$ to 0.72$)$ & 0.47 & 0.72 (0.07 to 7.14$)$ & 0.78 \\
\hline$\beta$-blocker & $0.90(0.34$ to 2.41$)$ & 0.84 & & 1.00 \\
\hline Calcium channel blocker & 1.64 (0.63 to 4.24$)$ & 0.31 & $6.90(0.71$ to 67.02$)$ & 0.10 \\
\hline \multicolumn{5}{|l|}{ Coronary angiogram: } \\
\hline Number of diseased vessels & $1.31(0.80$ to 2.14$)$ & 0.28 & $1.51(0.70$ to 3.31$)$ & 0.29 \\
\hline Presence of TCO $(\mathrm{y} / \mathrm{n})$ & 1.39 (0.52 to 3.69$)$ & 0.51 & $1.25(0.27$ to 5.75$)$ & 0.78 \\
\hline \multicolumn{5}{|l|}{ CMR before revascularisation: } \\
\hline $\operatorname{LVEF}(\%)$ & 0.99 (0.95 to 1.02$)$ & 0.37 & $1.01(0.96$ to 1.06$)$ & 0.70 \\
\hline LVEDVI (ml) & $1.02(1.00$ to 1.04$)$ & 0.03 & $1.01(0.98$ to 1.04$)$ & 0.30 \\
\hline LVESVI (ml) & $1.02(1.00$ to 1.04$)$ & 0.03 & $1.01(0.98$ to 1.05$)$ & 0.54 \\
\hline SVI (ml) & $1.01(0.97$ to 1.05$)$ & 0.61 & $1.02(0.96$ to 1.08$)$ & 0.54 \\
\hline LV mass index $(\mathrm{g})$ & $1.01(0.99$ to 1.04$)$ & 0.31 & $1.03(0.99$ to 1.07$)$ & 0.20 \\
\hline LV mass absolute $(\mathrm{g})$ & $1.01(1.00$ to 1.02$)$ & 0.17 & $1.01(1.00$ to 1.03$)$ & 0.15 \\
\hline Hyperenhancement (g) & $1.02(0.99$ to 1.05$)$ & 0.21 & $1.02(0.98$ to 1.07$)$ & 0.30 \\
\hline Presence of hyperenhancement $(y / n)$ & $1.33(0.60$ to 2.93$)$ & 0.48 & $1.66(0.50$ to 5.51$)$ & 0.41 \\
\hline \multicolumn{5}{|l|}{ CMR after revascularisation: } \\
\hline LVEF (\%) & $0.97(0.94$ to 1.00$)$ & 0.03 & $1.01(0.95$ to 1.06$)$ & 0.84 \\
\hline Change in LVEF pre/post (\%) & $0.96(0.92$ to 1.00$)$ & 0.06 & $0.99(0.92$ to 1.06$)$ & 0.75 \\
\hline LVEDVI (ml) & $1.02(1.00$ to 1.03$)$ & 0.13 & $0.99(0.96$ to 1.03$)$ & 0.74 \\
\hline LVESVI (ml) & $1.02(1.00$ to 1.04$)$ & 0.01 & $1.00(0.96$ to 1.04$)$ & 0.96 \\
\hline SVI (ml) & $0.98(0.94$ to 1.02$)$ & 0.31 & $0.98(0.92$ to 1.04$)$ & 0.46 \\
\hline LV mass index $(\mathrm{g})$ & $1.01(0.98$ to 1.03$)$ & 0.72 & $1.00(0.96$ to 1.05$)$ & 0.85 \\
\hline LV mass absolute (g) & $1.00(0.99$ to 1.02$)$ & 0.50 & $1.00(0.98$ to 1.02$)$ & 0.88 \\
\hline Hyperenhancement (g) & $1.03(1.00$ to 1.06$)$ & 0.03 & $1.03(0.99$ to 1.07$)$ & 0.10 \\
\hline Presence of hyperenhancement $(\mathrm{y} / \mathrm{n})$ & $2.46(0.93$ to 6.50$)$ & 0.07 & 5.95 (0.77 to 46.09$)$ & 0.09 \\
\hline New hyperenhancement $(\mathrm{g})$ & $1.12(1.05$ to 1.19$)$ & $<0.001$ & $1.10(1.00$ to 1.22$)$ & 0.05 \\
\hline Presence of new hyperenhancement $(y / n)$ & 3.05 (1.42 to 6.59$)$ & 0.004 & 2.99 (0.95 to 9.44$)$ & 0.06 \\
\hline
\end{tabular}

secondary endpoint. One patient with a non-fatal myocardial infarction also died during the observation period. All events occurred in patients with complete follow-up.

Procedure-related new hyperenhancement, when measured either as a continuous or as a categorical variable, was the strongest predictor of the primary outcome, followed by age, LV functional measures (LVESVI and LVEF) after revascularisation and dichotomised troponin values according to the ESC/ACC/ AHA/WHF guidelines, although the latter was not statistically significant $(p=0.08)$ (table 2$)$. Total hyperenhancement after revascularisation was also significantly associated with the primary endpoint, as were some LV functional measures (LVEDVI and LVESVI) before revascularisation. However, we did not observe any significant association between pre-existing hyperenhancement and the primary outcome in this cohort. Likewise, absolute troponin values and the change in LVEF after revascularisation were not significantly associated with occurrence of the primary outcome (table 2 ). 
Table 3 Independent predictors of the primary endpoint of death, non-fatal myocardial infarction, prolonged ventricular arrhythmia or hospitalisation with unstable angina or heart failure

\begin{tabular}{|c|c|c|}
\hline & HR $(95 \% \mathrm{Cl}) *$ & p Value \\
\hline \multicolumn{3}{|c|}{ Final model with new hyperenhancement as a categorical variable: } \\
\hline Presence of new hyperenhancement $(\mathrm{y} / \mathrm{n})$ & $3.11(1.43$ to 6.77$)$ & 0.004 \\
\hline \multicolumn{3}{|l|}{ Addition of extra variables: $\dagger$} \\
\hline LVEF after revascularisation (\%) & $0.98(0.95$ to 1.02$)$ & 0.31 \\
\hline Troponin after revascularisation $(\mu \mathrm{g} / \mathrm{l})$ & $1.02(0.96$ to 1.08$)$ & 0.52 \\
\hline Troponin after revascularisation $>0.2 \mu \mathrm{g} / \mathrm{t}$ & $1.26(0.33$ to 4.73$)$ & 0.73 \\
\hline PMI & $1.36(0.46$ to 4.03$)$ & 0.57 \\
\hline All other variables from table 2 & & $>0.10$ \\
\hline \multicolumn{3}{|c|}{ Final model with new hyperenhancement as a continuous variable: } \\
\hline New hyperenhancement $(\mathrm{g})$ & $1.10(1.03$ to 1.17$)$ & 0.005 \\
\hline \multicolumn{3}{|l|}{ Addition of extra variables: $\uparrow$} \\
\hline LVEF after revascularisation (\%) & $0.99(0.95$ to 1.02$)$ & 0.49 \\
\hline Troponin after revascularisation $(\mu \mathrm{g} / \mathrm{l})$ & $1.01(0.96$ to 1.07$)$ & 0.71 \\
\hline Troponin after revascularisation $>0.2 \mu \mathrm{g} / \mathrm{l}$ & $1.25(0.43$ to 3.61$)$ & 0.68 \\
\hline PMl & $1.58(0.58$ to 4.41$)$ & 0.88 \\
\hline All other variables from table 2 & & $>0.10$ \\
\hline
\end{tabular}

\footnotetext{
*All models were adjusted for age and sex.

$\dagger$ After inclusion of each of the extra variables, HR for new hyperenhancement remained $>2.6$ with $p$ value $<0.05$

$\vdots 0.2 \mu \mathrm{g} / \mathrm{l}$ equals the 99th centile of the lower limit of quantification.

-After inclusion of each of the extra variables, HR for new hyperenhancement (continuous) remained $>1.08$ with $p$ value $<0.05$.

LVEF, denotes left ventricular ejection fraction; PMI, procedure-related myocardial infarction according to the ESC/ACC/AHA/WHF guidelines.
}

When all significant variables from table 2 (with new hyperenhancement either as a categorical or as a continuous variable) were evaluated in a sex-adjusted and age-adjusted multiple Cox regression model, only new hyperenhancement remained significantly predictive of the outcome. Patients who had evidence of new hyperenhancement had a 3.1 fold higher risk of events ( $95 \%$ confidence interval (CI) 1.43 to 6.77) (table 3 and fig 1). We also observed a dose-response relation between the absolute magnitude of new hyperenhancement and the outcome with each gram of new myocardial injury translating into an excess risk of about $10 \%$ for the primary outcome $(95 \%$ CI $3 \%$ to $17 \%$ ).

In contrast, after allowing for new hyperenhancement, LVEF after revascularisation and dichotomised troponin values were no longer predictive of the primary outcome. Addition of troponin, or each of the other variables individually, did not

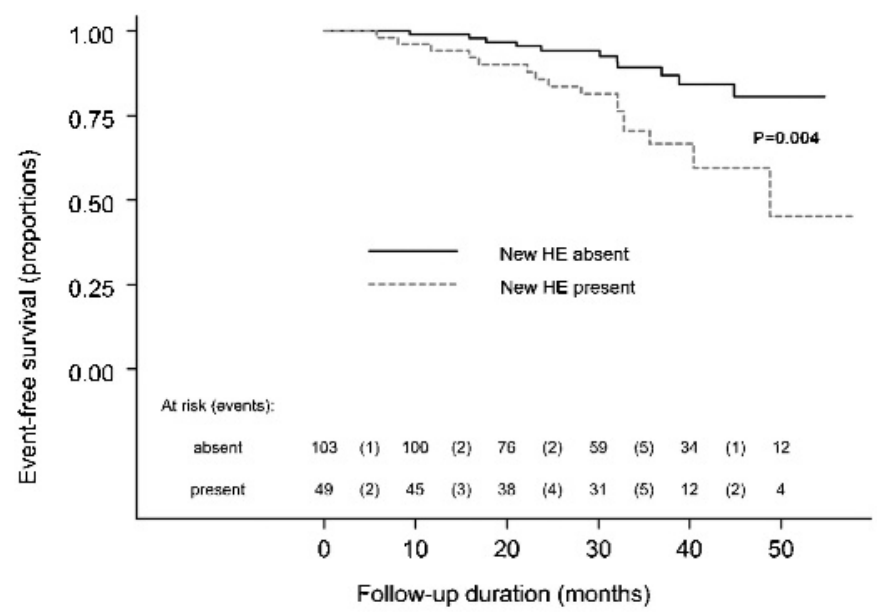

Figure 1 Age-adjusted and sex-adjusted Kaplan-Meier survival curves showing the proportion of patients remaining event-free for the primary endpoint of death, non-fatal myocardial infarction, ventricular arrhythmia or hospitalisation due to angina or worsening heart failure. $\mathrm{HE}$, hyperenhancement. reveal any other significant independent predictors, or eliminate the significant association between new hyperenhancement and the primary outcome (table 3).

With respect to the secondary outcome, only new hyperenhancement showed a significant association in the univariate Cox regression model (table 2 and fig 2). Thus, each gram of new myocardial necrosis was associated with an increased risk of death, non-fatal myocardial infarction or sustained ventricular arrhythmia of $10 \%$ (95\% confidence interval $0 \%$ to $22 \%$ ). Considering the primary endpoint, $81 \%$ of patient who had no new hyperenhancement remained event-free after 50 months, compared with only $45 \%$ of patients who had new HE, a difference of $36 \%$ (fig 1). Considering the secondary endpoint, the percentages remaining event-free after 50 months were $92 \%$ with no new hyperenhancement and $66 \%$ with new $\mathrm{HE}$, a difference of $26 \%$ (fig 2). Figures 3 and 4 illustrate two patient examples of new injury resulting in adverse clinical outcome.

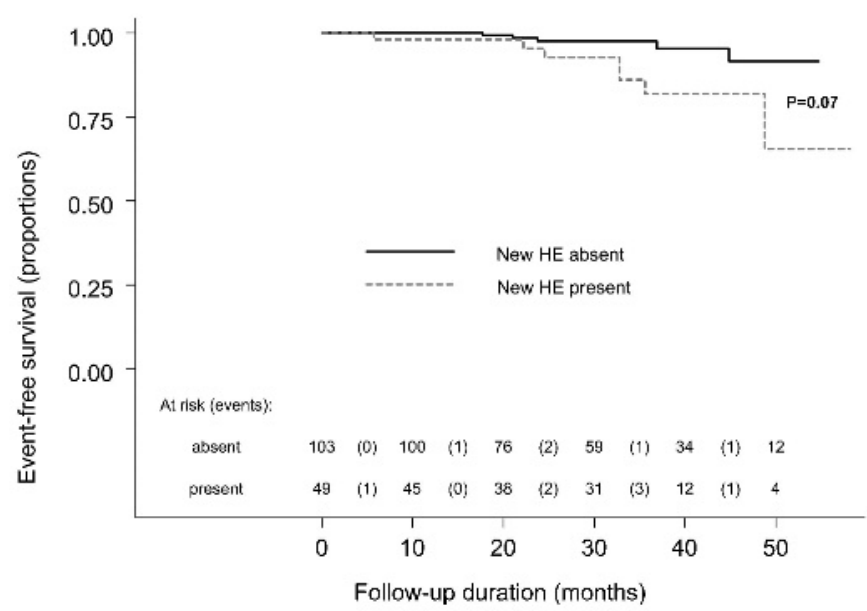

Figure 2 Age-adjusted and sex-adjusted Kaplan-Meier survival curves showing the proportion of patients remaining event-free for the secondary end point of death, non-fatal myocardial infarction or ventricular arrhythmia. $\mathrm{HE}$, hyperenhancement. 
Figure 3 Contrast-enhanced preoperative MRI scan in short axis plane (A) in a 76-year-old diabetic patient presenting with dyspnoea. He underwent CABG for severe three-vessel coronary artery disease. Postoperative short axis (B) contrast enhanced images show new inferior and infero-septal hyperenhancement (white arrow). He died of heart failure 12 months after surgery.
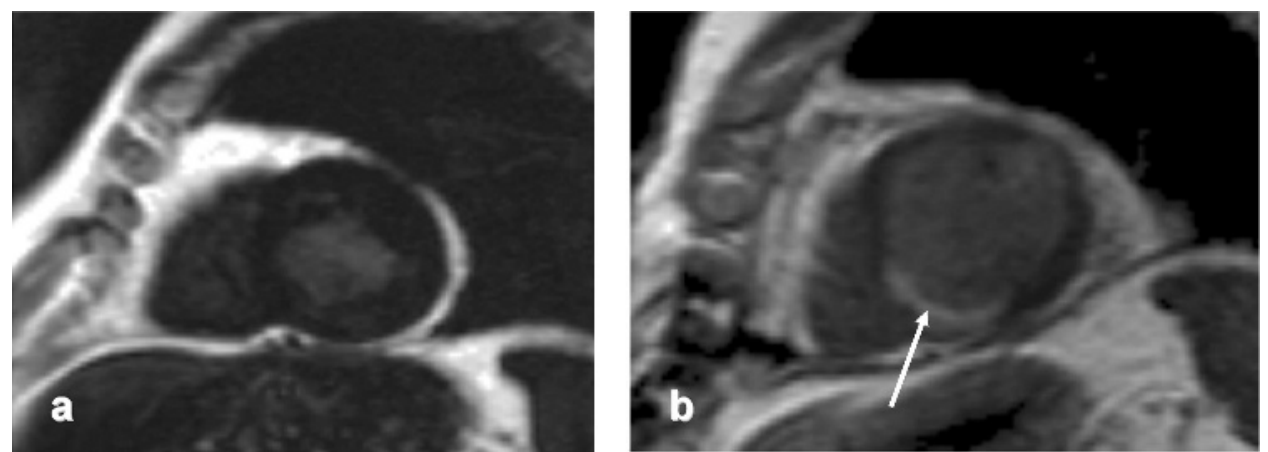

Since the study population consisted of patients undergoing either $\mathrm{CABG}$ or PCI, we assessed the potential modifying effect of type of revascularisation on the observed association between new myocardial injury and future adverse events. The analyses showed very similar hazard ratios for the two types of revascularisation both for the primary outcome (hazard ratio (HR) 3.29; 95\% CI 1.10 to 9.88 vs 2.98; 95\% CI 1.00 to 8.86 ; CABG vs PCI, respectively) and for the secondary outcome (HR $3.02 ; 95 \%$ CI 0.49 to 18.37 vs $3.40 ; 95 \%$ CI 0.76 to 15.19 ; CABG vs PCI, respectively).

\section{DISCUSSION}

In this study we demonstrate that, in patients with coronary artery disease undergoing elective coronary revascularisation, even small amounts of revascularisation-related myocardial necrosis identified by CMR have strong and independent prognostic values. Patients who had evidence of new myocardial hyperenhancement after coronary revascularisation had a threefold higher risk of the primary endpoint of death, nonfatal myocardial infarction or a major cardiovascular event compared to those without any evidence of new myonecrosis. The predictive value of new hyperenhancement was much stronger than that of established risk factors such as cardiac troponin levels or left ventricular dysfunction.

Cardiac biomarkers are readily available and thus have been widely used in previous studies that have evaluated the significance of post-procedural myocardial infarction. As stated above, however, there are several limitations inherent in the use of biomarkers in this setting that might explain why previous studies, with adequate control for potential confounders including baseline troponin values, showed a non-significant, ${ }^{21}$ or at most a mild, ${ }^{22}{ }^{23}$ association between post-procedural biomarker values and poorer outcomes. Measurements of global left ventricular function such as ejection fraction, on the other hand, are neither specific nor sensitive for detection of myocardial injury, as they can be affected by other factors such as loading conditions, myocardial ischaemia without necrosis (stunning), and remodelling. In particular, smaller myocardial tissue loss as is often encountered after revascularisation is less likely to translate into measurable changes in left ventricular size or function.

Direct visualisation and quantification of myocardial tissue loss with the use of DE-CMR offers several advantages over traditional clinical tools for the assessment of infarct size. For example, unlike the highly time-dependent kinetics of troponin assays, myocardial hyperenhancement-related to revascularisation injury persists over time $e^{15}$ and therefore allows a more accurate ascertainment of new myocardial tissue injury. This might explain why hyperenhancement appears to be a better predictor of future cardiac risk than procedural measures of troponin despite their relation demonstrated in this and previous studies. ${ }^{15}$

Our results support the concept of a direct relation between the magnitude of irreversible myocardial injury and the likelihood of adverse clinical outcomes. There are at least two potential explanations for this observation. First, as has been extensively debated previously, ${ }^{54}$ the occurrence of new myonecrosis might be a marker of a severe burden of coronary atherosclerosis and arterial inflammation. Of the 27 patients experiencing the primary endpoint, 13 patients (48\%) presented with heart failure, reflecting the link of myocardial tissue loss with systolic and/or diastolic heart failure. Second, any degree of irreversible myocardial injury may predispose to arrhythmias, which would influence survival even without a substantial reduction in global left ventricular function. Further validation of this concept is provided by a recent study in patients with a prior MI, showing that the extent of the peri-infarct zone characterised by CMR provides incremental prognostic value beyond left ventricular systolic volume index or ejection fraction. ${ }^{25}$

There is now compelling evidence to support the use of a revascularisation strategy in patients with acute coronary
Figure 4 Mid-ventricular short axis image $(A)$ in an 80-year-old patient presenting with increasing angina. Coronary angiography revealed severe two-vessel CAD (long right coronary artery (RCA) and left circumflex artery (LCX) lesions). The patient underwent apparently successful $\mathrm{PCl}$ of both vessels but contrast enhanced image in the same slice position post-PCI (B) reveals small subendocardial hyperenhancement of the inferior wall (arrow). He suddenly died 2 years after $\mathrm{PCl}$.
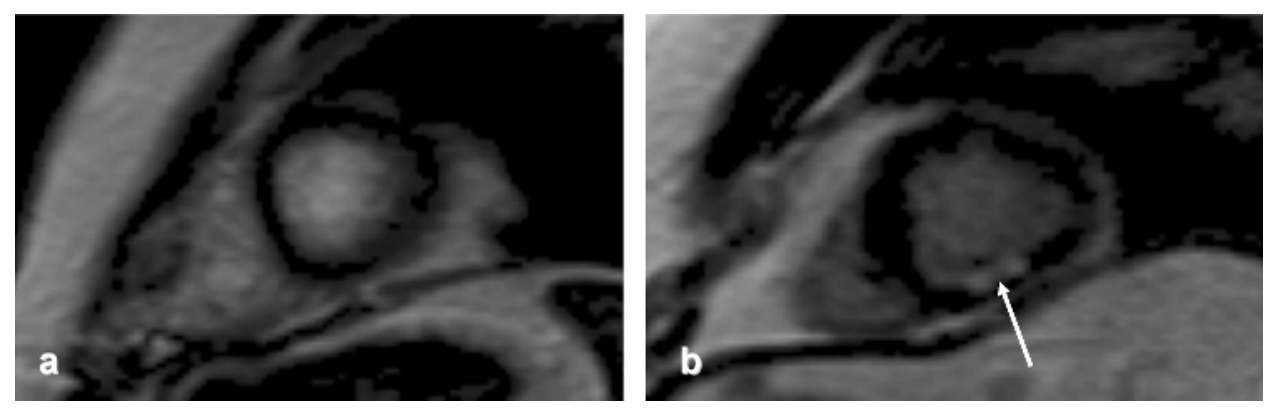
syndromes, particularly those with ST-elevation myocardial infarction or significant three-vessel coronary artery disease, who are at higher risk of adverse events. ${ }^{26-28}$ However, careful assessment of risk-benefit ratio remains crucial, particularly in those at low to moderate risk for future adverse events. This has been underscored by the results of the recent COURAGE clinical trial, which showed that use of PCI as the initial management strategy (in addition to optimal medical therapy) in patients with stable coronary artery disease did not reduce the risk of future coronary events when compared with optimal medical therapy alone. ${ }^{29}$ Our findings show that even small amounts of myocardial damage caused by the intervention itself might portend an adverse clinical outcome over the medium term. Hence, there is a "trade-off" between luminal gain from the revascularisation procedure versus the myonecrosis-related to the revascularisation.

\section{Limitations}

First, semi-automatic techniques, using full-width at halfmaximum (FWHM) criteria, have recently (following commencement of this study) been shown to correlate best with postmortem data for infarct sizing, ${ }^{30}$ and our use of the two standard deviation approach might have overestimated infarct size. However, this approach was consistent for both preprocedure and post-procedure CMR scans and hence would not have influenced our study's main conclusions. Second, we were unable to verify the cause of death in all patients. However, the pre-specified use of all-cause mortality would, if anything, have reduced the estimated hazard ratios and diluted their statistical significance. Finally, owing to the limited number of events and the observational design of the study, there remains a certain chance of the observed association being, at least partly, explained by other measured or unmeasured confounding factors.

In conclusion, irreversible myocardial injury can be reliably detected after elective coronary revascularisation using DECMR and injury detected in this way translates into an appreciable risk of adverse clinical events, even if the area involved is small. The increased risk of future clinical events should be taken into consideration by clinicians when patients are considered for coronary revascularisation. Particularly in patients with low overall risk, the competing risk of new myocardial damage caused by the intervention may well outweigh the benefit of revascularisation.

Funding: This work was supported by the British Heart Foundation (BHF), the UK Medical Research Council (MRC) and Cancer Research UK (CRUK). SN and APB are partially funded by the Oxford Biomedical Research Centre. The funding agencies had no influence on data analysis, their interpretation or publication of the results.

Competing interests: SN has received a research grant from Siemens.

Ethics approval: The study was approved by our institutional ethics committee and informed written consent was obtained from each patient.

Provenance and peer review: Not commissioned; externally peer reviewed.

\section{REFERENCES}

1. Januzzi JL, Lewandrowski K, MacGillivray TE, et al. A comparison of cardiac troponin $T$ and creatine kinase-MB for patient evaluation after cardiac surgery. $J$ Am Coll Cardiol 2002;39:1518-23.

2. Herrmann J. Peri-procedural myocardial injury: 2005 update. Eur Heart J 2005;26:2493-519

3. Thygesen K, Alpert JS, White HD. Universal definition of myocardial infarction. Eur Heart J 2007;28:2525-38.

4. Cutlip DE, Kuntz RE. Cardiac enzyme elevation after successful percutaneous coronary intervention is not an independent predictor of adverse outcomes. Circulation 2005;112:916-23.
5. Bhatt DL, Topol EJ. Does creatinine kinase-MB elevation after percutaneous coronary intervention predict outcomes in 2005? Periprocedural cardiac enzyme elevation predicts adverse outcomes. Circulation 2005;112:906-15; discussion 923

6. Eggers KM, Lagerqvist B, Venge $P$, et al. Persistent cardiac troponin I elevation in stabilized patients after an episode of acute coronary syndrome predicts long-term mortality. Circulation 2007:116:1907-14.

7. Jeremias A, Kleiman NS, Nassif D, et al. Prevalence and prognostic significance of preprocedural cardiac troponin elevation among patients with stable coronary artery disease undergoing percutaneous coronary intervention: results from the Evaluation of Drug Eluting Stents and Ischemic Events Registry. Circulation 2008;118:632-8.

8. Miller WL, Garratt KN, Burritt MF, et al. Timing of peak troponin T and creatine kinase-MB elevations after percutaneous coronary intervention. Chest 2004;125:275-80.

9. Antman EM, Morrow DA. Biomarker release after percutaneous coronary intervention: a message from the heart. Circ Cardiovasc Intervent 2008;1:3-6.

10. Wu E, Judd RM, Vargas JD, et al. Visualisation of presence, location, and transmural extent of healed 0 -wave and non-0-wave myocardial infarction. Lancet 2001:357:21-8.

11. Wagner A, Mahrholdt H, Holly TA, et al. Contrast-enhanced MRI and routine single photon emission computed tomography (SPECT) perfusion imaging for detection of subendocardial myocardial infarcts: an imaging study. Lancet 2003;361:374-9.

12. Wu KC, Zerhouni EA, Judd RM, et al. Prognostic significance of microvascula obstruction by magnetic resonance imaging in patients with acute myocardial infarction. Circulation 1998;97:765-72.

13. Kim RJ, Chen EL, Lima JA, et al. Myocardial Gd-DTPA kinetics determine MRI contrast enhancement and reflect the extent and severity of myocardial injury after acute reperfused infarction. Circulation 1996;94:3318-26.

14. Selvanayagam JB, Petersen SE, Francis JM, et al. Effects of off-pump versus onpump coronary surgery on reversible and irreversible myocardial injury: a randomized trial using cardiovascular magnetic resonance imaging and biochemical markers. Circulation 2004:109:345-50.

15. Selvanayagam JB, Porto I, Channon $\mathrm{K}$, et al. Troponin elevation after percutaneous coronary intervention directly represents the extent of irreversible myocardial injury insights from cardiovascular magnetic resonance imaging. Circulation 2005; 111:1027-32.

16. Ricciardi MJ, Wu E, Davidson CJ, et al. Visualization of discrete microinfarction after percutaneous coronary intervention associated with mild creatine kinase-MB elevation. Circulation 2001;103:2780-3.

17. Kwong RY, Chan AK, Brown KA, et al. Impact of unrecognized myocardial scar detected by cardiac magnetic resonance imaging on event-free survival in patients presenting with signs or symptoms of coronary artery disease. Circulation 2006:113:2733-43

18. Simonetti OP, Kim RJ, Fieno DS, et al. An improved MR imaging technique for the visualization of myocardial infarction. Radiology 2001;218:215-23.

19. Porto I, Selvanayagam JB, Van Gaal WJ, et al. Plaque volume and occurrence and location of periprocedural myocardial necrosis after percutaneous coronary intervention: insights from delayed-enhancement magnetic resonance imaging, thrombolysis in myocardial infarction myocardial perfusion grade analysis, and intravascular ultrasound. Circulation 2006;114:662-9.

20. Kao JT, Wong IL, Lee JY, et al. Comparison of Abbott AxSYM, Behring Opus Plus, DPC Immulite and Ortho-Clinical Diagnostics Vitros ECi for measurement of cardiac troponin I. Ann Clin Biochem 2001;38(Pt 2):140-6.

21. Miller WL, Garratt KN, Burritt MF, et al. Baseline troponin level: key to understanding the importance of post-PCl troponin elevations. Eur Heart J 2006;27:1061-9.

22. Prasad A, Singh M, Lerman A, et al. Isolated elevation in troponin T after percutaneous coronary intervention is associated with higher long-term mortality. J Am Coll Cardiol 2006;48:1765-70.

23. Prasad A, Rihal CS, Lennon RJ, et al. Significance of periprocedural myonecrosis on outcomes after percutaneous coronary intervention: an analysis of preintervention and postintervention troponin T levels in 5487 patients. Circ Cardiovasc Intervent 2008;1:10-9.

24. Mehran R, Dangas G, Mintz GS, et al. Atherosclerotic plaque burden and CK-MB enzyme elevation after coronary interventions: intravascular ultrasound study of 2256 patients. Circulation 2000;101:604-10.

25. Yan AT, Shayne AJ, Brown KA, et al. Characterization of the peri-infarct zone by contrast-enhanced cardiac magnetic resonance imaging is a powerful predictor of post-myocardial infarction mortality. Circulation 2006;114:32-9.

26. Cannon CP, Weintraub WS, Demopoulos LA, et al. Comparison of early invasive and conservative strategies in patients with unstable coronary syndromes treated with the glycoprotein Ilb/llla inhibitor tirofiban. N Engl J Med 2001;344:1879-87.

27. Fox KA, Poole-Wilson PA, Henderson RA, et al. Interventional versus conservative treatment for patients with unstable angina or non-ST-elevation myocardial infarction: the British Heart Foundation RITA 3 randomised trial. Randomized Intervention Trial of unstable Angina. Lancet 2002;360:743-51.

28. Mehta SR, Cannon CP, Fox KA, et al. Routine vs selective invasive strategies in patients with acute coronary syndromes: a collaborative meta-analysis of randomized trials. JAMA 2005;293:2908-17.

29. Boden WE, O'Rourke RA, Teo KK, et al. Optimal medical therapy with or without PCI for stable coronary disease. N Engl J Med 2007;356:1503-16.

30. Amado LC, Gerber BL, Gupta SN, et al. Accurate and objective infarct sizing by contrast-enhanced magnetic resonance imaging in a canine myocardial infarction model. J Am Coll Cardiol 2004;44:2383-9. 